\title{
Preservation of entanglement in a two-qubit-spin coupled system
}

\author{
$\mathrm{Yu}-\mathrm{Chen} \mathrm{Hou}$ \\ School of Electronic and Information Engineering, Beihang University, Xueyuan Road No. 37, Beijing 100191, \\ PR China \\ Guo-Feng Zhang \\ School of Physics and Nuclear Energy Engineering, Beihang University, Xueyuan Road No. 37, Beijing \\ 100191, PR China \\ Yan Chen \\ School of Physics and Nuclear Energy Engineering, Beihang University, Xueyuan Road No. 37, Beijing \\ 100191, PR China \\ Heng Fan \\ Beijing National Laboratory for Condensed Matter Physics, Institute of Physics, Chinese Academy of Sciences, \\ Beijing 100190, PR China
}

\begin{abstract}
A theoretical scheme to preserve the entanglement in a two-qubit-spin coupled system in the presence of Dzyaloshinskii-Moriya (DM) anisotropic antisymmetric interaction is proposed. Based on a sequence of operations performed periodically on the system, the scheme can preserve the entanglement of the system starting from any initial state with any high precision for any long duration.
\end{abstract}

PACS:03. 67. Lx, 03. 65. Ta

Keywords: Preservation, Entanglement, Concurrence

\section{Introduction}

Entanglement plays a central role in quantum information processing. However, entanglement is so fragile that in real circumstances it cannot be easily preserved. Therefore, the implementation of preservation of entanglement is a crucial element in the realization of quantum information processing. Last decade has seen a number of theories, and techniques on the preservation of

Email address: Corresponding author: gf1978zhang@buaa.edu.cn (Guo-Feng Zhang) 
entanglement, most of which are based on formalized mathematics models and quantum optical systems. Some examples are error-avoiding [1], bang-bang control [2] and Super-Zeno effect [3]. The well-known Quantum Zeno effect, which was first presented in the literature as a paradoxical consequence of measurements on quantum mechanics [4], is also a useful tool for quantum state protection [5], entanglement control [6] and entanglement preservation [7]. But quantum optical systems, like their classical counterparts, have an intrinsic disadvantage: poor scalability. Among other schemes that have been proposed to construct quantum information systems, the ones based on solid state systems are believed to have the best scalability [8]. Moreover, the solid state schemes can largely take advantage of modern semiconductor technology and micro-fabrication technology. Because of these advantages, the study of solid state quantum computation is currently an important field [9, 10, 11, 12, 13]. Solid spin systems are among those solid-based candidates for the realization of the entanglement. The spin chains not only have useful applications such as the quantum state transfer, but also display rich entanglement features [14, 15]. The Heisenberg chain, the simplest spin chain, has been used to construct a quantum computer and quantum dots $[9 \mid$. By suitable coding, the Heisenberg interaction alone can be used for quantum computation [12, 16, 17]. Obviously, researches on the preservation of entanglement in spin systems will provide a possibility of an ideal physical foundation for quantum information systems. The objective of the present work is to construct a scheme to control the evolution of a two-qubit-spin system (a typical case of a general spin system) and preserve entanglement. In this paper, the scheme is described first and then an example is given to illustrate the effect of the scheme.

\section{Model and scheme}

In this paper, we consider the Heisenberg model with DM interaction which can be written as

$$
H=J_{1} \sigma_{1 x} \sigma_{2 x}+J_{2} \sigma_{1 y} \sigma_{2 y}+D\left(\sigma_{1 x} \sigma_{2 y}-\sigma_{1 y} \sigma_{2 x}\right)
$$

where $\sigma_{m n}(m=1,2 ; n=x, y)$ 's are Pauli matrices, $J_{i}(i=1,2)$ 's are the real coupling coefficients and $D$ is the DM vector coupling which is assumed only along $z$ axis. The DM anisotropic antisymmetric interaction arises from spin-orbit coupling [18, 19, 20, 21]. We can control the system by interrupting the free evolution periodically with a certain operation, so that the state 
of the controlled evolution is confined in a subset of a state space very close to the initial state and the entanglement can be preserved very well. This idea is inspired by prior work of R. Rossi 22].

The free evolution $U_{f}$ governed by the Hamiltonian is:

$$
U_{f}(t)=e^{-i H t / \hbar}
$$

In order to preserve the initial entanglement, we can interrupt the free evolution before the state deviates from the initial state too much, and guide the state back to its starting point. We call this process the cyclic evolution. The implementation of such a process is given as follows.

The cyclic evolution $U_{c T}$ is constructed in this way: the system evolves freely for some time $T$, then a certain operation $O$ is applied to the system, then a same evolution and a same operation, which can be written as

$$
U_{c T}(\tau)= \begin{cases}U_{f}(0) \equiv I & \tau=0 \\ U_{f}(\tau) & 0<\tau<T \\ U_{f}(T) & \tau \rightarrow T_{-} \\ O U_{f}(T) & \tau \rightarrow T_{+} \\ U_{f}(\tau-T) O U_{f}(T) & T<\tau<2 T \\ U_{f}(T) O U_{f}(T) & \tau \rightarrow(2 T)_{-} \\ O U_{f}(T) O U_{f}(T) \equiv I & \tau \rightarrow(2 T)_{+}\end{cases}
$$

where $O=I_{1} \otimes \sigma_{2 z}$. Note that the cyclic evolution is discontinuous for two points, $\tau=T$ and $\tau=2 T$, where operations are applied. These discontinuities are demonstrated explicitly above by one-sided limits with - and + signs indexing the below (left) limit and above (right) limit respectively. Pay special attention to the equation $O U_{f}(T) O U_{f}(T) \equiv I(\forall T)$, which can be proved by simple calculations. This equation shows that the evolution is indeed a cyclic evolution (with period 2T), at the end of that the state is guided back to the starting point. Note the graduality of the free evolution at the initial state:

$$
\lim _{T \rightarrow 0} U_{f}(T)=I
$$


which suggests that the state will not deviate from the starting point very fast. So we can expect to preserve any initial state to any desired precision in such a cycle, by shortening the time interval $T$ to a sufficient level. This will be presented explicitly in Eq.(11) and Fig.2.

High precision of the preservation requires the time interval $T$ to be short. Meanwhile, we want the preservation lasts as long as possible. We can manage to achieve both of these two points (high precision and long duration) by fully taking advantage of the cyclic character of the cyclic evolution. In fact, the controlled evolution is implemented by repeating the same cyclic evolution (interrupting the free evolution with the same operation in period $2 T$ ):

$$
\begin{aligned}
& U_{c}(t) \\
= & U_{c T}(\tau) O U_{f}(T) O U_{f}(T) \cdots O U_{f}(T) O U_{f}(T) \\
= & U_{c T}(\tau) I \cdots I \\
= & U_{c T}(\tau),
\end{aligned}
$$

where $\tau=t-2 k T$ and $k=\left\lfloor\frac{t}{2 T}\right\rfloor$ is the number of complete cycles ( \lfloor\rfloor is the floor operator) so that $t=\tau+2 k T$ and $0 \leq \tau<2 T$. In this way, the preservation can last forever, theoretically.

The construction of the scheme to control the evolution is now completed. Notably, there are no restrictions on the precision, the duration or the initial state.

\section{Example}

To illustrate the effect of the scheme, we will compare the entanglement of the free evolution and that of the controlled one in an example, where the initial state is assumed to be in a Bell state: $|\psi(0)\rangle=\frac{1}{\sqrt{2}}|01\rangle+\frac{1}{\sqrt{2}}|10\rangle$ and the entanglement is calculated by concurrence [23]:

$$
C(t)=\max \left(0,2 \max \left\{\sqrt{\mu_{i}}\right\}-\sum_{i} \sqrt{\mu_{i}}\right),
$$

where $\mu_{i}$ 's are eigenvalues of the operator $\rho S \rho^{*} S$ with $S=\sigma_{1 y} \otimes \sigma_{2 y}$ and $\rho=U|\psi(0)\rangle\langle\psi(0)| U^{\dagger}$.

Applying Eq.(2), Eq.(3) and Eq.(5) to equation Eq.(6), we obtain the concurrences of the free, cyclic and controlled evolutions and their minimums:

$$
C_{f}(t)=\frac{\sqrt{2 D^{2} \cos \left(4 t \sqrt{K^{2}+4 D^{2}}\right)+K^{2}+2 D^{2}}}{\sqrt{K^{2}+4 D^{2}}},
$$




$$
\begin{gathered}
C_{c}(t)=C_{c T}(\tau)=\left\{\begin{array}{ll}
\frac{\sqrt{2 D^{2} \cos \left(4 \tau \sqrt{K^{2}+4 D^{2}}\right)+K^{2}+2 D^{2}}}{\sqrt{K^{2}+4 D^{2}}} & 0 \leq \tau \leq T \\
\frac{\sqrt{2 D^{2} \cos \left(\sqrt{K^{2}+4 D^{2}}(8 T-4 \tau)\right)+K^{2}+2 D^{2}}}{\sqrt{K^{2}+4 D^{2}}} & T \leq \tau \leq 2 T
\end{array},\right. \\
\left(C_{f}(t)\right)_{\min }=\frac{|K|}{\sqrt{K^{2}+4 D^{2}}}, \\
\left(C_{c}(t)\right)_{\min }=\left(C_{c T}(\tau)\right)_{\min }=\frac{\sqrt{2 D^{2} \cos \left(4 \sqrt{K^{2}+4 D^{2}} T\right)+K^{2}+2 D^{2}}}{\sqrt{K^{2}+4 D^{2}}},
\end{gathered}
$$

where $K=J_{1}+J_{2}, \tau=t-2 k T$ and $k=\left\lfloor\frac{t}{2 T}\right\rfloor$ is the number of complete cycles. Note the precision of the preservation that can be achieved:

$$
\lim _{T \rightarrow 0}\left(\left(C_{c}(t)\right)_{\min }\right)=1
$$

which indicates that the initial entanglement is preserved well by shortening the time interval $T$, as we have expected in Eq.(4).

The effect of the scheme can be illustrated better by Fig.1, where a comparison of concurrences of the free and the controlled evolution is given. The concurrence of free evolution vibrates severely, while that of the controlled one is kept at a high level close to the initial concurrence. The controlled evolution is composed of a sequence of cyclic evolutions. Every cyclic evolution has two stages: the first one is a falling stage and the second one is a rising stage. At the end of each stage, an operation is performed: the first operation terminates the falling stage and initializes the rising stage, while the second operation restores the initial state and ensures a smooth transition from the current cyclic evolution to the next one. Therefore, the concurrence is kept from deviating too far away by a sequence of periodic interruptions.

As is mentioned above in Eq.(4) and Eq.(11), the requirement of high precision preservation can be satisfied by increasing the frequency (shortening the time interval $T$ ) of the sequence of interruptions, which is illustrated in Fig.2.

\section{Conclusions}

We have proposed a theoretical scheme to preserve the entanglement in a two-qubit-spin system with DM interaction by applying a sequence of operations periodically on the system. This scheme can preserve the entanglement of the system starting from any initial state with any high precision for any long duration. 


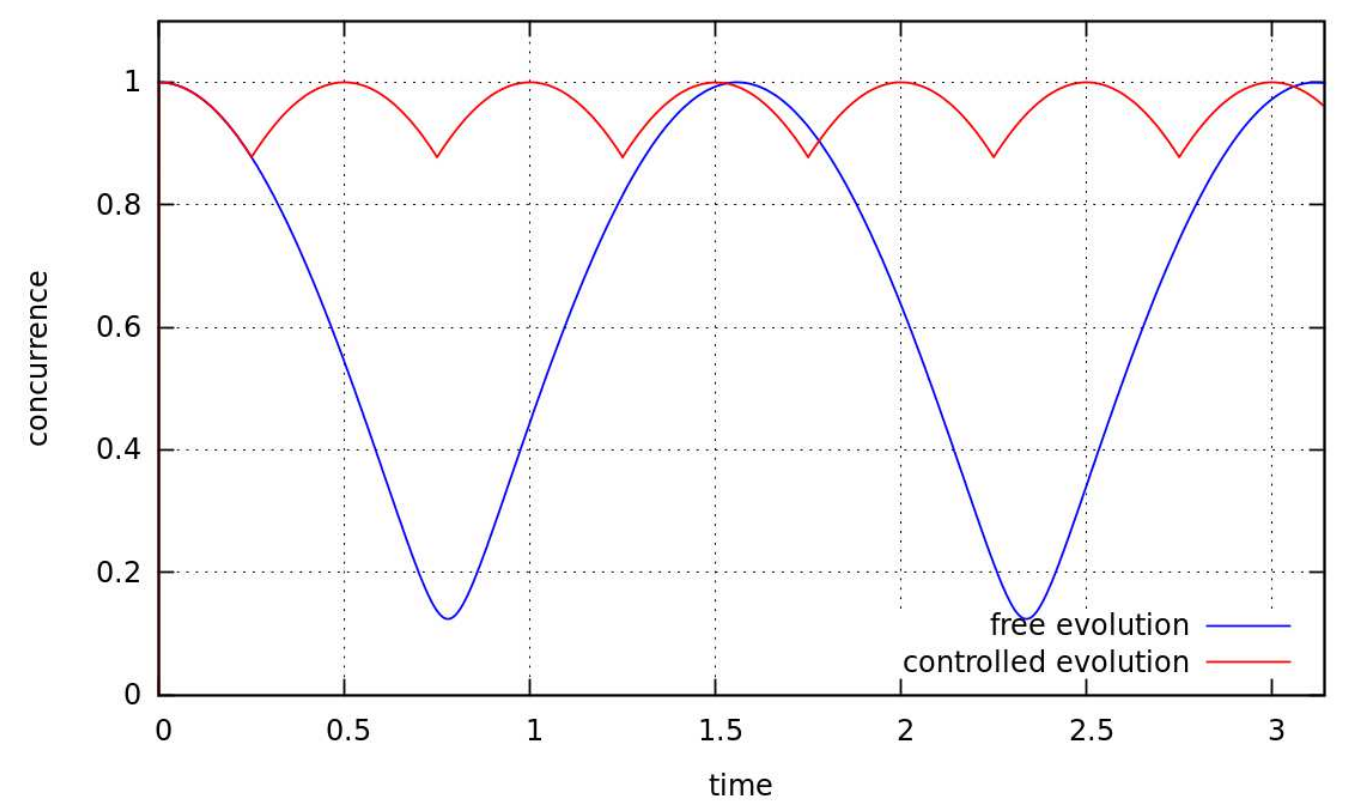

Figure 1: (color online) Comparison of concurrences with parameters $T=1 / 4, D=1 / 2$ and $K=1 / 8$.

\section{Acknowledgements}

This work was supported by the National Science Foundation of China under Grants No. 10874013. Heng Fan acknowledges the support of the National Science Foundation of China under Grant No. 10974247. Thanks to the folks at Beihang University: Shisheng Zhang at school of physics provided revising assist; Yanlong Hou at school of electric engineering and Hua Tang at school of electronic engineering provided software suggestions.

\section{References}

[1] C. P. Yang, S. Chu and S. Y. Han, Phys. Rev. A 66, 034301 (2002).

[2] L. Viola and S. Lloyd, Phys. Rev. A 58, 2733 (1998).

[3] D. Dhar, L. K. Grover and S. M. Roy, Phys. Rev. Lett. 96, 100405 (2006).

[4] B. Misra, E. C. G. Sudarshan, J. Math. Phys. 18, 756 (1977).

[5] P. Facchi and S. Pascazio, Phys. Rev. Lett. 89, 080401 (2002).

[6] J. G. Oliveira Jr, R. Rossi Jr and M. C. Nemes, Phys. Rev. A 78, 044301 (2008) . 


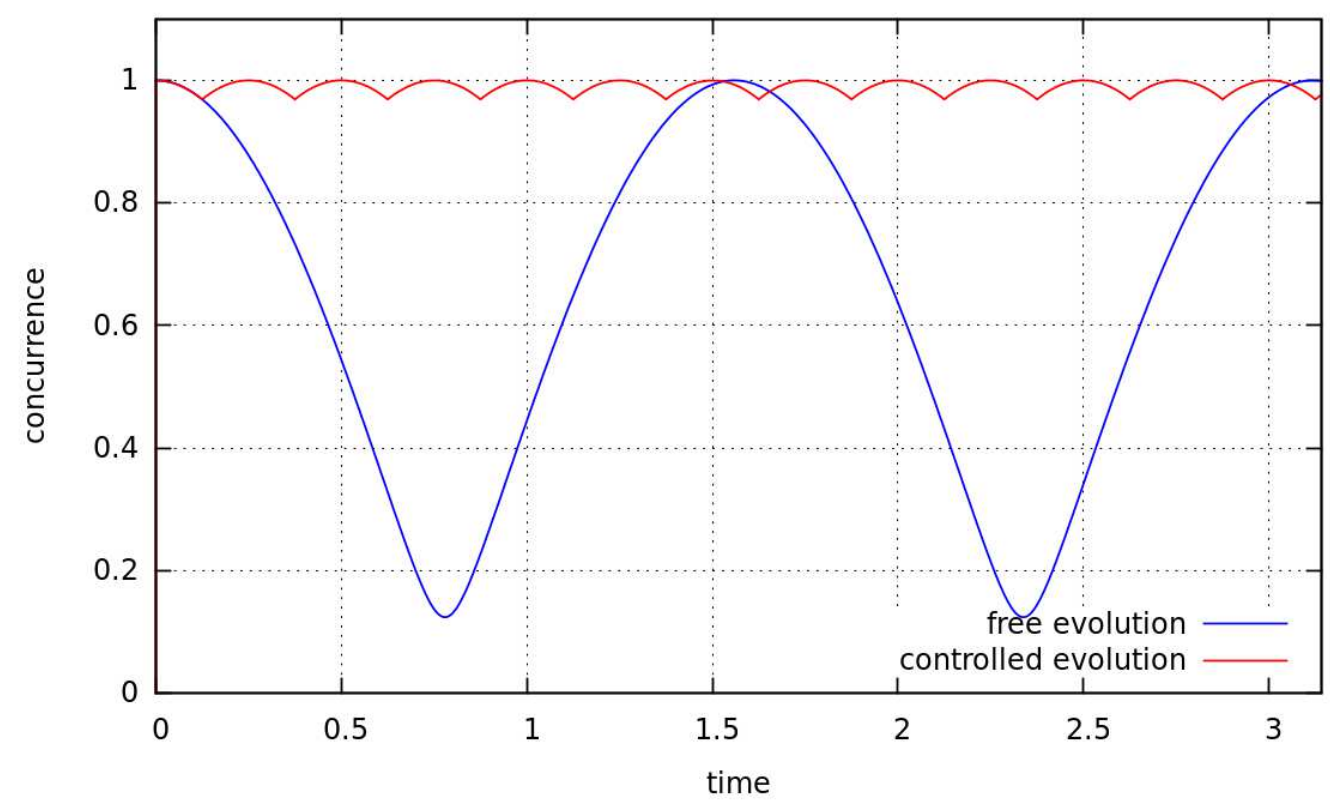

Figure 2: (color online) Comparison of concurrences (higher operation frequency) with parameters $T=1 / 8$, $D=1 / 2$ and $K=1 / 8$.

[7] S. Maniscalco, F. Francica, R. L. Zaffino, N. L. Gullo and F. Plastina, Phys. Rev. Lett. 100, $090503(2008)$.

[8] J. L. O’Brien, S. R. Schofield, M. Y. Simmons, R. G. Clark, A. S. Dzurak, N. J. Curson, B. E. Kane, N. S. McAlpine, M. E. Hawley, and G. W. Brown, Phys. Rev. B 64, 161401 (2001).

[9] D. Loss and D. P. DiVincenzo, Phys. Rev. A 57, 120 (1998).

[10] B. E. Kane, Nature 393, 133 (1998).

[11] G. Burkard, D. Loss and D. P. DiVincenzo, Phys. Rev. B 59, 2070 (1999).

[12] L. F. Santos and M. I. Dykman, Phys. Rev. B 68, 214410 (2003).

[13] J. Levy, Phys. Rev. A 64, 052306 (2001).

[14] S. Bose, Phys. Rev. Lett. 91, 207901 (2003).

[15] M. Christandl, N. Datta, A. Ekert, and A. J. Landahl, Phys. Rev. Lett.92, 187902 (2004). 
[16] D. A. Lidar, D. Bacon and K. B. Whaley, Phys. Rev. Lett. 82, 4556 (1999).

[17] D. P. Divincenzo, D. Bacon, J. Kempe, G. Burkard and K. B. Whaley, Nature 408, 339 (2000).

[18] I. Dzyaloshinskii, J. Phys. Chem. Solids 4, 241 (1958).

[19] T. Moriya, Phys. Rev. Lett. 4, 228 (1960).

[20] T. Moriya, Phys. Rev. 117, 635 (1960).

[21] T. Moriya, Phys. Rev. 120, 91 (1960).

[22] R. Rossi Jr, Phys. Lett. A 374, 2331 (2010).

[23] S. Hill and W. K. Wootters, Phys. Rev. Lett. 78, 5022 (1997). 